\title{
Semi-analytical Study of a One-dimensional Contaminant Flow in a Finite Medium
}

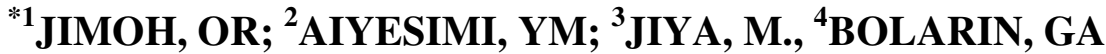 \\ ${ }^{1,2,3,4}$ Department of Mathematics, Federal University of Technology, Minna, Nigeria. \\ *Corresponding author E-mail: razaq.jimoh@futminna.edu.ng
}

\begin{abstract}
The Bubnov-Galerkin weighted residual method was used to solve a onedimensional contaminant flow problem in this paper. The governing equation of the contaminant flow, which is characterized by advection, dispersion and adsorption was discretized and solved to obtain the semi-analytical solution. The adsorption isotherm was assumed to be of Freudlich type. The results obtained were expressed in graphical form to show the effect of change in the parameters on the concentration of the contaminants. From the analysis of the results, it was discovered that the contaminant concentration decreases with increase in the distance from the origin as the dispersion and velocity coefficient decrease.
\end{abstract}

(C) JASEM

https://dx.doi.org/10.4314/jasem.v21i3.9

Keywords: Adsorption, advection, Bubnov-Galerkin weighted residuals, contaminant, dispersion

Contaminant transport in soil, groundwater and surface water has been in hydro-geological research history for many years. This is largely due to increased awareness of significant contamination of groundwater and surface water by industrial and human activities such as agricultural chemicals, accidental spills, landfills and buried hazardous materials. While agricultural chemicals are generally useful in the surface of the soil, their penetration into the vadose zone and groundwater could contaminate groundwater.

Groundwater in its natural state is generally of excellent quality because the physical structure and mineral constituents of rock have facility for purifying water. Before the establishment of industries, the major contaminants in groundwater came from viruses and bacteria. The presence of these microbiological organisms like bacteria, viruses and parasites in groundwater constitute some threat to our community health.

The transport equation which models the movement of contaminants through groundwater and surface water environments was reported by Bear (1997). These equations describe advection, diffusion and interaction with the solid matrix through which the water flow. They are often advection-dominated and require a lot of care when solved numerically. In order to predict the contaminant migration in the geological formation more accurately, a tasking job emerges for scientists. The job involves defining the flow lines of groundwater of the aquifers and the travel time of water along the flow lines.
Most researchers are of the view that the flow in the solute transport or contaminant flow model is predominantly horizontal as found in Bear (1997). Further research by Brainard and Gelhar (1991) discovered that appreciable vertical flow components do occur in the domain of vertically penetrating wells and streams.

The efforts in providing solutions to the contaminant flow problems were not in vain as lot of successes were achieved by some researchers. Okedayo and Aiyesimi (2005) studied the influence of retardation factor on the nonlinear contaminant flow problem. Okedayo et al., (2011) worked on the 1-Dimensional nonlinear contaminant transport equation with an initial and instantaneous point source. Their investigation revealed that the contaminant concentration decreases with increase in the distance from the origin. On the dispersion of solute, Ramakanta and Mehta (2010) explored the effect of longitudinal dispersion of miscible fluid flow through porous media.

The analytical solution to temporally dependent dispersion through semi-infinite homogeneous porous media by Laplace transform technique (LTT) was obtained by Yadav et al., (2011). Aiyesimi and Jimoh $(2012,2013)$ embarked on computational analysis of 1dimensional non-linear contaminant flow problem with an initial continuous point source using homotopy perturbation method. Their findings revealed that the concentration decreases with increase in time and distance from the origin for the non-reactive case. In this research, we provide a semi-analytical solution of the one-dimensional nonlinear contaminant flow 
problem in a finite medium using the Bubnov-Galerkin weighted residual method.

\section{MATERIALS AND METHODS}

Formulation of the Model: We consider an incompressible fluid flow through a finite homogeneous porous media with constant initial concentration in the transport domain. The flow is assumed to be in $\mathrm{x}$ direction only. The source concentration is assumed at the origin (i.e. at $x=0$ ). We assume that there is no solute flux at the end of the boundary. Let $C(x, t)$

$$
R \frac{\partial C(x, t)}{\partial t}=\frac{\partial}{\partial x}\left(D \frac{\partial C(x, t)}{\partial x}+U C(x, t)\right)
$$

where

$$
R=1+\frac{\rho_{d} k_{d}}{n}
$$

$k_{d}$ is the distribution coefficient which is defined as ratio of the concentration of adsorbed contaminant to that of dissolved contaminant. $\rho_{d}$ is the weight of dry unit of soil. It is assumed that (i) the fluid density and viscosity are constant, (ii) there was no adsorption in the flow, i.e., $k_{d}=0$.

From the assumptions above, equation (1) becomes

$$
\frac{\partial C(x, t)}{\partial t}=\frac{\partial}{\partial x}\left(D \frac{\partial C(x, t)}{\partial x}+U C(x, t)\right)
$$

We choose the following boundary and initial conditions

$$
\left.\begin{array}{l}
C(0, t)=C_{i} \\
C(1, t)=0 \\
C(x, 0)=1-x^{3}
\end{array}\right\}
$$

where $C_{0}$ is the initial contaminant concentration in the flow.

Method of Solution: Bubnov-Galerkin Method: Galerkin method is one of the weighted residual methods which is used in solving differential equations. When the problem at hand is an ordinary differential equation, we call the method, Galerkin weighted residual method and it requires only one equation residual. If the problem is a partial differential equation, the method is a BubnovGalerkin method and requires more than one equation residual. The method of weighted residual requires two types of functions namely, the basic functions and weight functions. The former is used to construct the trial solution while the latter is used as criterion to minimize the residual. In applying Bubnov-Galerkin method, the trial solution is chosen to satisfy the boundary conditions while the basic functions must satisfy the homogeneous boundary conditions. In particular, the basic functions are chosen as the weight functions.

In solving the above problem (3), we apply the Galerkin weighted residual method precisely the Bubnov-Galerkin method. The basic functions are chosen to satisfy the homogeneous boundary conditions as in Ames (1972), Edward (1972), Finlayson (1972) and Kythe et al., (1997). Equations (3) and (4) can be rewritten as 
$\left.\begin{array}{l}\frac{\partial C}{\partial t}=D \frac{\partial^{2} C}{\partial x^{2}}-U \frac{\partial C}{\partial x} \\ C(0, t)=C_{0} \\ C(1, t)=0 \\ C(x, 0)=1-x^{3}\end{array}\right\}$

The trial solution is assumed of the form:

$C_{T}(x, t)=\phi_{0}(x)+\sum_{n=1}^{2} A_{n}(t) \phi_{n}(x)$

where,

$\phi_{0}(x)=C_{0}(1-x)$

And

$\phi_{n}(x)=x^{n}(1-x)$

where $\phi_{n}(x)$ are the basis functions. The trial approximation is

$C_{T}(x, t)=C_{0}(1-x)+A_{1}(t) x(1-x)+A_{2}(t) x^{2}(1-x)$

The equation residual is

$R_{E}=\frac{\partial C}{\partial t}-D \frac{\partial^{2} C}{\partial x^{2}}+U \frac{\partial C}{\partial x}$

We substitute (9) into (5) and obtain

$R_{E}=A_{1}^{\prime}(t)\left(x-x^{2}\right)+A_{2}{ }^{\prime}(t)\left(x^{2}-x^{3}\right)-D\left((2-6 x) A_{2}{ }^{\prime}(t)-2 A_{1}(t)\right)$

$+U\left(A_{1}(t)(1-2 x)+A_{2}(t)\left(2 x-3 x^{2}\right)-C_{0}\right)$

The residual is minimized as follows:

$\int_{0}^{1} R_{E} \times w_{i}(x) d x=0$

where $w_{i}(x)$ are the weight functions are the same as the basis functions. i.e., $w_{1}=\left(x-x^{2}\right)$ and $w_{2}=\left(x^{2}-x^{3}\right)$ respectively.

On evaluating using the first weight function, i.e.,

$\int_{0}^{1} R_{E} \times w_{1}(x) d x=0$

we obtain

$\frac{1}{30} A_{1}{ }^{\prime}(t)+\frac{1}{60} A_{2}{ }^{\prime}(t)+\frac{D}{3} A_{1}(t)+\left(\frac{D}{6}+\frac{U}{60}\right) A_{2}(t)=\frac{U C_{0}}{6}$

Similarly,

$\int_{0}^{1} R_{E} \times w_{2}(x) d x=0$ gives

$\frac{1}{60} A_{1}{ }^{\prime}(t)+\frac{1}{105} A_{2}{ }^{\prime}(t)+\left(\frac{D}{6}-\frac{U}{60}\right) A_{1}(t)+\frac{2 D}{15} A_{2}(t)=\frac{U C_{0}}{12}$ 
The initial equation residual is

$R_{I}=C_{0}(1-x)+A_{1}(0) x(1-x)+A_{2}(0)\left(x^{2}-x^{3}\right)-\left(1-x^{3}\right)$

The initial residual equation is minimized as follows:

$\int_{0}^{1} R_{I} \times w_{i}(x) d x=0$

i.e.,

$\frac{1}{30} A_{1}(0)+\frac{1}{60} A_{2}(0)=-\left(\frac{C_{0}}{12}-\frac{2}{15}\right)$

and

$\frac{1}{60} A_{1}(0)+\frac{1}{105} A_{2}(0)=\frac{5}{84}-\frac{C_{0}}{30}$

From equations (18) and (19), the initial conditions of equations (14) and (15) are obtained as $A_{1}(0)=7-6 C_{0}$ and $A_{2}(0)=-6+7 C_{0}$ respectively.

Equations (14) and ( 15) are rearranged and gives the following equations:

$A_{1}{ }^{\prime}(t)=-(7 U+10 D) A_{1}(t)+(16 D-4 U) A_{2}(t)+5 U C_{0}$

$A_{2}{ }^{\prime}(t)=14 U A_{1}(t)+(7 U-42 D) A_{2}(t)=0$

Equations (20) and (21) can be rewritten in matrix notation as

$\left(\begin{array}{l}A_{1}{ }^{\prime}(t) \\ A_{2}{ }^{\prime}(t)\end{array}\right)=\left(\begin{array}{cc}-p & q \\ r & s\end{array}\right)\left(\begin{array}{l}A_{1}(t) \\ A_{2}(t)\end{array}\right)+\left(\begin{array}{l}z \\ 0\end{array}\right)$

The parameters in the equation (22) are as defined below.

$p=7 U+10 D, q=16 D-4 U, r=14 U, s=7 U-42 D$ and $z=5 U C_{0}$.

Equation (22) is solved with the initial conditions $A_{1}(0)$ and $A_{2}(0)$ above using the eigenvalues and eigenvectors method and obtained the following results:

$$
\begin{aligned}
A_{1}(t)= & a_{1} q \exp \left(-\frac{p}{2}+\frac{s}{2}+\frac{1}{2} k\right) t-a_{2} q \exp \left(-\frac{p}{2}+\frac{s}{2}-\frac{1}{2} k\right) t+\frac{\frac{p q z}{2}+\frac{q s z}{2}-\frac{q z k}{2}}{q k\left(-\frac{p}{2}+\frac{s}{2}+\frac{1}{2} k\right)} \\
& -\frac{\frac{p z q}{2}+\frac{p s z}{2}+\frac{q z k}{2}}{q k\left(-\frac{p}{2}+\frac{s}{2}-\frac{1}{2} k\right)} \\
A_{2}(t)= & a_{1}\left(\frac{p}{2}+\frac{s}{2}+\frac{1}{2} k\right) \exp \left(-\frac{p}{2}+\frac{s}{2}+\frac{1}{2} k\right) t+a_{2}\left(-\frac{p}{2}-\frac{s}{2}+\frac{1}{2} k\right) \exp \left(-\frac{p}{2}+\frac{s}{2}-\frac{1}{2} k\right) t \\
& \left(\frac{p z}{2}+\frac{s z}{2}-\frac{z}{2} k\right)\left(\frac{p}{2}+\frac{s}{2}+\frac{1}{2} k\right) \\
+ & \frac{\left(\frac{p z}{2}+\frac{s z}{2}+\frac{z}{2} k\right)\left(-\frac{p}{2}-\frac{s}{2}+\frac{1}{2} k\right)}{q k\left(-\frac{p}{2}+\frac{s}{2}-\frac{1}{2} k\right)}
\end{aligned}
$$

By applying the initial conditions on the equations (23) and (24) and solving the resulting equations simultaneously, the values of $a_{1}$ and $a_{2}$ are obtained as 


$$
\begin{aligned}
a_{1}= & \frac{-\frac{z s p}{2}-\frac{z s^{2}}{2}+\frac{z s k}{2}+\frac{p^{3} z}{4}+\frac{p^{2} s z}{4}-\frac{s^{2} z}{4}-\frac{s^{3} z}{4}-\frac{p z k^{2}}{4}+\frac{z s k^{2}}{4}}{q k^{2}\left(-\frac{p}{2}+\frac{s}{2}+\frac{1}{2} k\right)\left(-\frac{p}{2}+\frac{s}{2}-\frac{1}{2} k\right)} \\
& +\frac{\left(7-6 C_{0}\right)\left(-\frac{p}{2}-\frac{s}{2}+\frac{1}{2} k\right)+q\left(-7+6 C_{0}\right)}{q k} \\
a_{2}= & \frac{-\frac{z s^{2} p}{4}+\frac{p^{3} z}{4}+\frac{p^{2} s z}{4}-\frac{s^{3} z}{4}-\frac{p z k^{2}}{4}+\frac{z s k^{2}}{4}-z s\left(\frac{p}{2}+\frac{s}{2}+\frac{1}{2} k\right)}{q k\left(-\frac{p}{2}+\frac{s}{2}+\frac{1}{2} k\right)\left(-\frac{p}{2}+\frac{s}{2}-\frac{1}{2} k\right)} \\
& +\frac{q\left(-7+6 C_{0}\right)-\left(7-6 C_{0}\right)\left(\frac{p}{2}+\frac{s}{2}+\frac{1}{2} k\right)}{q k}
\end{aligned}
$$

where

$$
k=\sqrt{(p+s)^{2}+4 r q} \text {. }
$$

The semi-analytical solution of the contaminant flow problem (3) is obtained by substituting equation (23) and (24) in equation (9).

\section{RESULTS AND DISCUSSION}

The semi-analytical solution obtained is used to study the behavior of the contaminant in the flow for values of $C=10, D=0.7,0.8,0.9$ in figure 1 and $3, D=1.0,1.1,1.2$ in figure $2, U=0.1,0.5,0.9$ in figure 4.

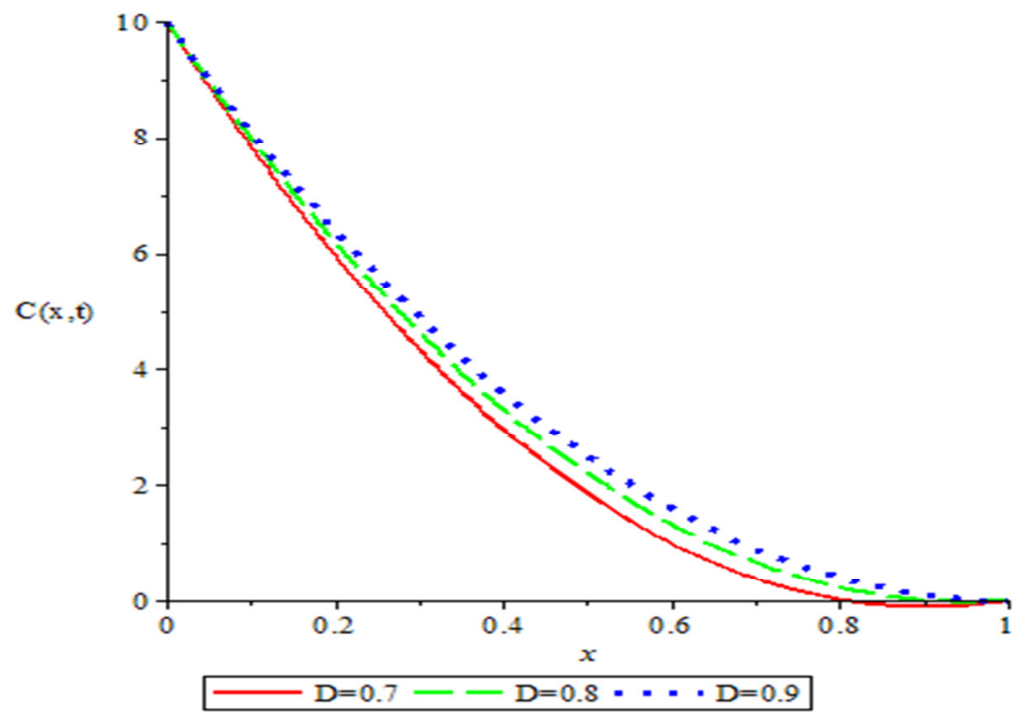

Fig 1: The concentration profile of the contaminant concentration with distance for $C_{0}=10, U=0.1, t=0.1$. 


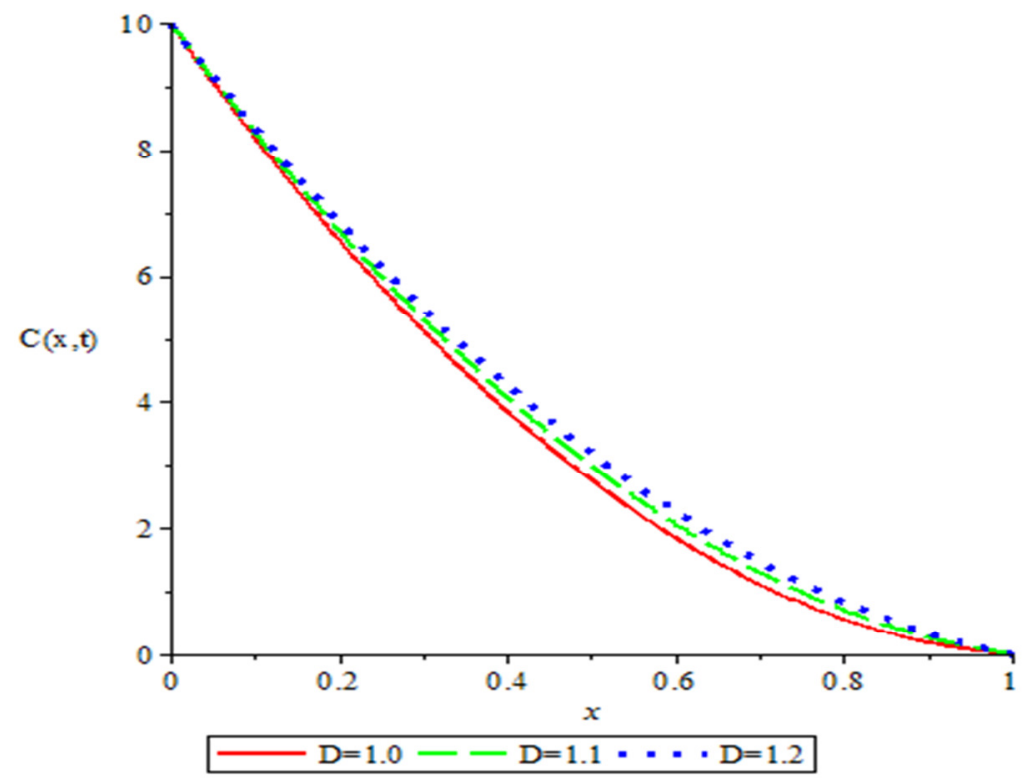

Fig 2: The concentration profile of the contaminant concentration with distance for

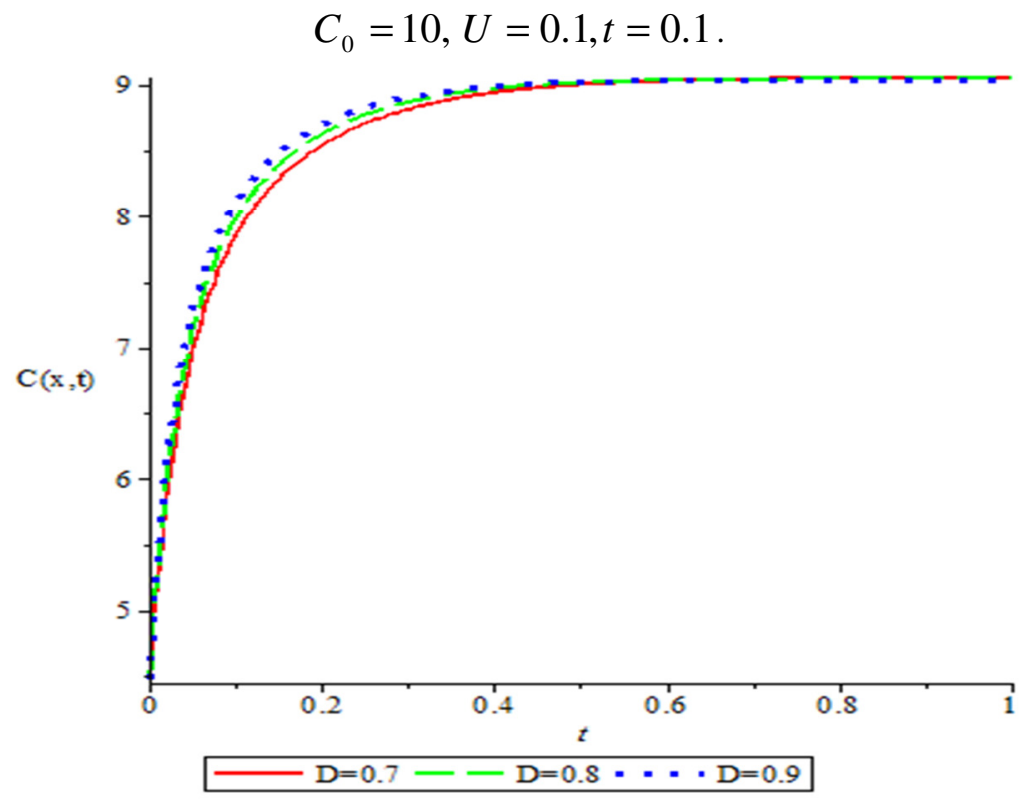

Fig 3: The concentration profile of the contaminant concentration with time for $C_{0}=10, U=0.1, x=0.1$. 


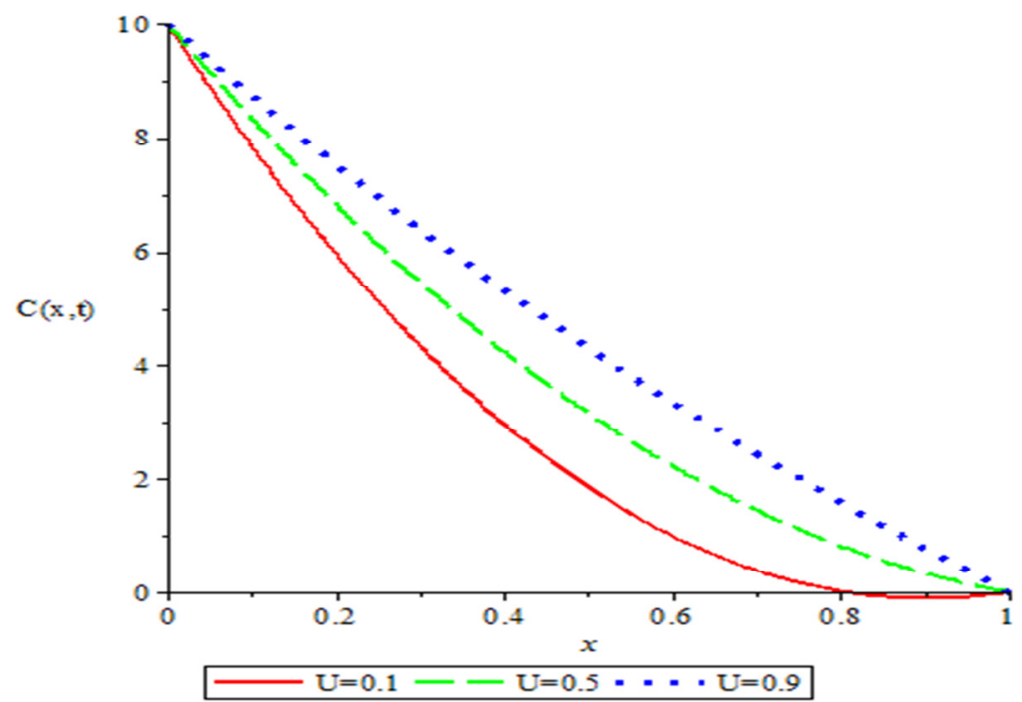

Fig 4: The concentration profile of the contaminant concentration with distance for

$$
C_{0}=10, D=0.7, t=0.1 \text {. }
$$

Figures 1 and 2 show the contaminant concentration profile with distance for varying dispersion coefficient. The graphs show that the contaminant concentration decreases as the dispersion coefficient decreases. Figure 3 is the contaminant concentration profile with distance for varying dispersion coefficient. It shows that as the dispersion coefficient increases, the contaminant concentration increases with time. Findings from figure 4 also reveal that as the velocity component decreases, the contaminant concentration decreases.

The semi-analytical solution obtained for the onedimensional contaminant flow in this research is in line with the one-dimensional flow in real system. This is evident from the figures 1,2 and 4 above. As the contaminant travels with the flowing water, the concentration decreases as the distance covered increases.

Conclusion: A contaminant flow model with constant initial concentration is solved to predict the contaminant concentration along transient groundwater in a finite homogeneous medium by Bubnov-Galerkin method. The dispersion and velocity coefficients are varied to study the contaminant behavior along the flow. Our findings reveal that the contaminant concentration decreases along the spatial direction as the dispersion $D$ and the velocity $U$ coefficients decline.

\section{REFERENCES}

Aiyesimi, Y. M; Jimoh, O. R. (2012). Computational Analysis of 1-Dimensional non-linear reactive contaminant flow problem with an initial continuous point source. Journal of Nigerian Association of Mathematical Physics. 22: 543543

Aiyesimi, Y. M; Jimoh, O. R. (2013). Analytical solution of non-linear contaminant Flow problems with initial continuous point source by homotopy perturbation method. Nigerian Journal of Technological Research. 8(1): 43-46.

Ames, W. F. (1972). Numerical Methods for Partial Differential Equations. Academic Press. New York. 1: 320-332

Bear, J. (1997). Hydraulics of Groundwater. McGraw-Hill. New York.

Brainard, E. C; Gelhar, L. W. (1991). Influence of vertical flow on groundwater transport. Groundwater. 2915: 693-701.

Edward, L. B. (1972). A look at some method of solving partial differential equations and eigenvalue problems. National Technical Information service. Springfield.

Finlayson, B. A. (1972). The method of weighted residuals and variational principles. Academic press. New York.

Freezer, R. A; Cherry, J.A. (1979). Groundwater. N. J. Prentice Hall, Englewood Cliffs Inc. 
Kythe, P. K; Puri, P; Schaferkotter, M. R. (1997). Partial differential equations and Mathemtica. CRC Press Inc., Florida.

Okedayo, T. G; Aiyesimi, Y. M. (2005). Influence of retardation factor on the non-linear contaminant flow. The Journal of Education. 4:27-32.

Okedayo,T.G; Owoloko, E. A; Osafile, O.E. (2011). A regular perturbation analysis of thenon-linear contaminant transport equation with initial and instantaneous point source. Australia Journal of Basic and Applied Sciences. 5(8):1273-1277.

Ramakanta, M; Mehta, M. N. (2010). Effect of longitudinal dispersion of miscible fluid flow through porous media. Advanced theoretical and Applied Mechanics. 3(5):211-220.
Singh, M. K; Mahato, N. K; Kumar, N. (2015). Pollutant's horizontal dispersion along and against

Sinusoidally varying velocity from a pulse type source. Acta Geophysica, 63(1), 214-261.

Yadav, R. R; Jaiswal, D. K; Yadav, H. K. (2011). Temporary dependent dispersion through semiinfinite homogeneous porous media: an analytical solution. IJRRAS. 6(2): 158-163. 